Von Alexandra Dehnhardt

m jahrelangen Kampf um den Erhalt der Elbe als einem der letzten frei fließenden Flüsse mit unschätzbarem ökologischen Wert ist endlich ein Durchbruch erzielt worden: Umweltverbände und -experten haben allen Grund zu feiern. So bekennt sich die Bundesregierung in ihrer Koalitionsvereinbarung zu einem Verzicht auf die einst in der Bundesverkehrswegeplanung festgeschriebene Umsetzung weiterer Ausbaumaßnahmen an der Elbe und den Bau von Staustufen an der Saale. Die ablehnende Haltung der Bundesregierung gegenüber den Flussbaumaßnahmen ist jedoch nicht das Ergebnis eines grundsätzlich veränderten Leitbildes für Stromlandschaften, sondern eher das Resultat der Notwendigkeit schnellen Handelns, hier im Sinne einer kurzfristigen Gefahrenabwehr und Risikominimierung infolge der Hochwasserkatastrophe.

Im Rahmen der Flusskonferenz, die als unmittelbare Reaktion auf die Hochwasserkatastrophe im September stattfand, wurde ein Fünf-Punkte-Programm zur Verbesserung des vorbeugenden Hochwasserschutzes beschlossen, dessen zügige Realisierung ebenfalls im Koalitionsvertrag festgeschrieben ist. Doch genau dieser Passus könnte unter Umständen sogar eine Hintertür für eine Aufweichung der Ausbaustoppbeschlïsse darstellen, wenn der politische Handlungsdruck infolge einer wieder geringer werdenden Sensibilität der Öffentlichkeit für Hochwassergefahren abnehmen sollte und überkommene Ansprüche an die Schiffbarkeit der Elbe wieder mehr und mehr in den Vordergrund rücken - letztlich sieht man sich hier erheblichen ökonomischen Interessen gegenüber. Denn bei genauerem Blick in das Programm stellt man fest, dass darin (nur) eine Neubewertung der Ausbauplanungen in ihren Auswirkungen auf den Hochwasserschutz vorgesehen war; die Ergebnisse sollen entsprechend in die Erstellung des neuen Bundesverkehrswegeplans Anfang 2003 einfließen.

Den Stopp der Ausbauplanungen allein mit dem Hochwasserrisiko zu begründen, wird ohnehin nicht gelingen. Denn diese sind zwar für das Hochwassergeschehen relevant, aber nicht unbedingt von prioritärer Bedeutung. Der Ausbaustopp lässt sich viel eher aus der geringen ökonomischen Sinnhaftigkeit verbunden mit großen ökologischen Schäden des Elbeausbaus ableiten: Die im Rahmen der Bundesverkehrswegeplanung ursprünglich vorgesehenen Flussbaumaßnahmen beruhen auf einer Prognose des Güterverkehrs
Der rotgrüne Ausbaustopp von Elbe und Saale kann nur der Anfang sein

\section{Politik im Fluss?}

\section{Als historische Wende in der Fluss-} politik wurde kürzlich der im rot-grünen Koalitionsvertrag vereinbarte Ausbaustopp an Elbe und Saale gefeiert. Doch noch ist offen, ob mit dieser zunächst öffentlichkeitswirksamen Maßnahme tatsächlich auch ein daverhaftes Umdenken im Umgang mit Flüssen in Richtung eines nachhaltigen Managements stattgefunden hat.

von 1992, die sich längst als falsch erwiesen hat. Dem gegenüber steht eine Gefährdung der Auenlandschaften, die aufgrund von Nutzen-KostenKalkülen, die den ökologischen Wert der Landschaft einbeziehen, keineswegs gerechtfertigt ist (1).

Auch wenn die Willenserklärung der Bundesregierung zum Ausbaustopp dauerhaft Bestand hat, geht es in der Sache also um mehr: Eine Weichenstellung für ein grundsätzliches Umdenken im Umgang mit Flüssen ist angezeigt, und Flussbaumaßnahmen und Hochwasserschutz sind im Kontext einer integrierten Konzept- und Leitbildentwicklung für Flusslandschaften zu begreifen. Positive Ansätze in dieser Richtung sind im FünfPunkte-Programm der Bundesregierung bereits zu finden. So wird beispielsweise das Ausmaß der Flutkatastrophe in den Kontext der Auswirkungen menschlichen Handelns gestellt und die Notwendigkeit eines konsequenten Klimaschutzes hervorgehoben. Auch geht das Hochwasserschutzprogramm in seiner Konzeption über Sofortmaßnahmen zur Gefahrenabwehr und einen rein additiven Hochwasserschutz hinaus. Betont wird die Bedeutung von natürlichen Überschwemmungsflächen, Gewässerrenaturierungen und des dezentralen Wasserrückhaltes im Einzugsgebiet von Flüssen.

\section{Fehlende Konkretisierung}

Dem Programm fehlt es jedoch noch weitgehend an inhaltlichen und konzeptionellen Konkretisierungen. Ohne diese laufen die Planungen Gefahr, reine politische Willenserklärungen zu bleiben.
Laut Bundesregierung wird 2004 die nächste internationale Flusskonferenz stattfinden. Bis dahin muss eine grundsätzliche Wende in der Flusspolitik auf den Weg gebracht werden. Die wesentliche Aufgabe für Politik und Forschung besteht für die nächsten Jahre darin, Inhalte und Richtung dieser neuen Flusspolitik zu gestalten und integrierte Handlungskonzepte zur Umsetzung zu erarbeiten. Gerade an der Elbe besteht das Potenzial, eine dauerhafte ökonomische Entwicklung in Einklang mit der Erhaltung und Entwicklung natürlicher Funktionen und der Einzigartigkeit von Auenlandschaften zu bringen. Dazu müssen allerdings auch die damit verbundenen wirtschaftlichen Potenziale beispielsweise im Tourismus eröffnet werden - hier ist der neue Superminister Stolpe gefragt.

Zunächst bedarf es zum einen einer zeitnahen Umsetzung konkreter Maßnahmen wie die seit langem geforderte Rückverlegung von Deichen, Reaktivierung von Altauen und Entsiegelung von Uferbereichen. Zum anderen müssen die verschiedenen ökologischen, sozialen und ökonomischen Ansprüche an Flussgebiete abgewogen und in einem Gesamtkonzept integriert werden, um langfristige Lösungen zu erreichen. Im Einklang mit dem für Deutschland neuen Politikansatz des Flusseinzugsgebietsmanagements muss dazu nicht nur das Wissen um die komplexen ökologisch-ökonomischen Zusammenhänge und Entstehungsursachen von Hochwasserkatastrophen vertieft werden. Auch sollten alle Anspruchsgruppen in einen kontinuierlichen und kooperativen Prozess eingebunden werden, um eine gemeinsame Vorstellung eines Leitbildes für die Elbe zu entwickeln. Nur dann wird die Elbe tatsächlich - wie es Umweltverbände fordern - zum Symbol für eine neue Flusspolitik.

\section{Anmerkung}

(1) Vgl. hierzu z.B. Meyerhoff, J./ Dehnhardt, A.: Nachhaltige Entwicklung der Elbe. In: Ökologisches Wirtschaften, Nr. 5/2002, S. 27-28.

\section{Die Autorin}

Alexandra Dehnhardt ist wissenschaftliche Mitarbeiterin im Forschungsfeld Umweltökonomie und -politik des Instituts für ökologische Wirtschaftsforschung.

Kontakt: IÖW, Potsdamer Str. 105, 10785 Berlin. Tel. 030/884594-27, Fax 030/ 8825439,

E-Mail: Alexandra.Dehnhardt@ioew.de 
(c) 20I0 Authors; licensee IÖW and oekom verlag. This is an article distributed under the terms of the Creative Commons Attribution Non-Commercial No Derivates License (http://creativecommons.org/licenses/by-nc-nd/3.o/), which permits unrestricted use, distribution, and reproduction in any medium, provided the original work is properly cited. 